\title{
The Poggendorff and its variants do arouse the same perceptual process
}

\author{
DARIUS C. MacKAY and P. L. NEWBIGGING \\ McMaster University, Hamilton, Ontario L8S 4K1, Canada
}

\begin{abstract}
The first two of five experiments reported show that the complete Poggendorff and three of its variants all produce the same illusory effect and that, for all four patterns, that effect is increased as the angle of interception of the obliques decreases and as the separation of the parallels increases. The third experiment shows that the differences in the magnitude of illusion produced by the variants is most probably due to the components retained in a particular variant and not to the simple reduction in the total amount of illusion pattern retained, which also correlates with magnitude of illusion. The final two experiments show that equal or greater positive transfer to the complete pattern is obtained from the variants as from the complete pattern after equal amounts of practice. The conclusion is reached that the complete pattern and the variants arouse the same single perceptual process. The data do not permit identification of that process.
\end{abstract}

It is appealing to suppose that an analysis of the Poggendorff illusion into its component parts will reveal those components that are critical in producing the apparent displacement from collinearity of the intercepting transversals, which constitutes the illusion, and so reveal the cause of this effect. Blix (1902) appears to have been the first to take this approach, and in his lengthy paper he presents and discusses many of the incomplete variants that have been the subject of recent experimental studies (e.g., Day, 1973a, b; Goldstein \& Weintraub, 1972; Pressey \& Sweeney, 1969; Weintraub \& Krantz, 1971).

While data on the major variants of the Poggendorff are now numerous, no clear empirical picture can be assembled. This is because of conflicting experimental outcomes from different laboratories. For example, in patterns containing only acute angles (see Figure 1c), it has been found that: (a) a negative (reversed) illusion occurs (Blix, 1902; Restle, 1969), (b) a negligible or no illusion occurs (Green \& Hoyle, 1964; Krantz \& Weintraub, 1973; Weintraub \& Krantz, 1971), and (c) a reduced but significant positive illusion occurs (Day, 1973b; Pressey \& Sweeney, 1972). Discrepant results have been reported for other variants of the illusion as well.

There are a number of possible reasons for these discrepant results, and prominent among them is the fact that different investigators have used widely different values of two critical variables in the display: the angle at which the oblique lines intercept the parallels and the extent by which the parallels themselves are separated. The effect of these two

This research was supported by Grant A-0175 from the National Research Council of Canada to P. L. Newbigging. variables, that the illusion increases regularly as angle of interception decreases and as the separation of the parallels increases, is well documented (Burmester, 1896; Velinsky, 1925). In addition, displays have differed in size and in the nature of the surrounding field; modes of presentation have differed as have psychophysical procedures. How these variations or combinations of them affect the magnitude of the illusion observed is difficult to say.

Against this background, and accepting that a quantitative comparison of the major variants of the Poggendorff might give some insight into the cause of the illusion, the first two experiments reported in this paper were designed to answer three main questions. They are: (1) Do the major variants produce the same illusory effect as the complete Poggendorff? (2) Do the variants respond in the same way as the complete pattern to manipulation of angle of interception and separation of the parallels? (3) Do individuals respond consistently to the variants? That is, will the illusion scores of individuals on the different patterns correlate positively? If the answer to all three of these questions is "yes," then there would seem to be some basis for assuming that the variants were arousing the same perceptual process.

\section{EXPERIMENT 1}

In this experiment, the angle at which the oblique line intercepts the parallel was varied in each of three incomplete Poggendorff patterns and the complete pattern, all shown in Figure 1.

\section{Method}

Stimuli. Each of the four patterns shown in Figure 1 was drawn in each of five versions. The versions differed in terms of the angle at which the oblique intercepted the parallel. The 


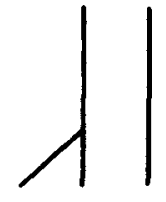

$\boldsymbol{A}$

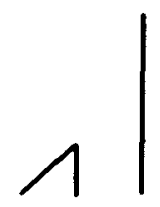

C

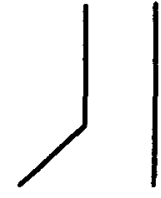

B

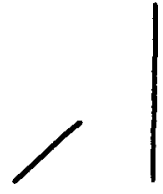

D
Figure 1. Patterns used in Experiments 1 and 2. (A) complete, (B) obtuse, (C) acute, (D) oblique.

angles used were $15^{\circ}, 30^{\circ}, 45^{\circ}, 60^{\circ}$, and $75^{\circ}$. The patterns were drawn on standard Ditto paper with the parallels $18 \mathrm{~cm}$ long and the oblique lines $3.5 \mathrm{~cm}$ in length. The parallels were separated by $3 \mathrm{~cm}$. In this way, a total of 20 (4 patterns $X 5$ angles) different patterns were prepared. Three copies of each of these patterns were bound in a haphazard order in a booklet. Each page containing one of the patterns was separated from adjacent patterns by a blank page of opaque blue paper so that the subject could see only one pattern at a time as he worked through the booklet.

Subjects. The subjects were six men and four women undergraduate students, between the ages of 18 and 22 , who were paid for their participation.

Procedure. The subjects were tested in a single group. They were seated at tables and provided with a booklet containing the illusion pattern and instructed that their task was to visually project the left-hand oblique and to draw, on signal, a clear mark on the right-hand parallel at the point where the oblique would meet it if it were, in fact, extended. They were told that only one choice could be made on a trial and that projecting the oblique by arm movements was not permitted. This procedure was illustrated with a Poggendorff figure drawn on the blackboard.

A yellow dot was placed on the table convenient to the subject's preferred hand. The subjects were instructed to keep their hands on the dot between trials. A trial was begun by the experimenter saying "turn." The subjects then turned the blue sheet to expose one of the Poggendorff patterns. After $10 \mathrm{sec}$, the experimenter said "now," and on this signal the subjects removed their preferred hands from the yellow dot and made their mark. This done, they turned the pattern over, exposing the next blue sheet. This procedure was continued until all 60 patterns had been completed, the whole session lasting about $40 \mathrm{~min}$.

\section{Results}

The data are the average differences between where the subjects made their marks and true collinearity. A Poggendorff illusion effect is observed when the subject places his mark below the point of true collinearity. The group data are shown in Figure 2. A two-factor repeated measures (Myers, 1972) analysis of variance of these data showed a significant effect of angle $[F(4,36)=3.81, p<.025]$, of pattern $[F(3,27)=8.49, p<.001]$, and a significant Angle by Pattern interaction $[\mathrm{F}(12,108)=2.84$, $p<.011$. The nature of this interaction is apparent in the graph, and it is clear that angle size has a smaller effect on the acute and oblique patterns than it does on the other two, although for these two an illusory effect significantly greater than zero is observed with the smaller angles.

Since a within-subjects design was used, it was possible to intercorrelate the illusion scores obtained on the four different patterns. These correlations had an average value of +0.80 and ranged from +0.71 $(p<.02)$ to $+0.88(p<.005)$.

\section{EXPERIMENT 2}

In this experiment, the separation of the parallel lines was varied in each of the incomplete Poggendorff patterns and the complete pattern, all shown in Figure 1.

\section{Method}

Stimuli. The stimuli were identical to those used in Experiment 1 , except that the angle of interception of the oblique lines was fixed at $30^{\circ}$ and the separation of the parallels was varied. The values of the separations used were $1,2,3,4$, and $5 \mathrm{~cm}$.

Subjects. The subjects were five men and five women undergraduate students, between the ages of 18 and 21 , who were paid for their participation.

Procedure. The procedure was identical in all respects to that used in Experiment 1.

\section{Results and Discussion}

The results of this experiment are shown in Figure 3. An analysis of variance of these data showed a significant effect of separation $[\mathrm{F}(4,36)=$ $5.88, p<.001]$, of pattern $[F(3,27)=42.70, p<.001]$ and a significant Separation by Pattern interaction

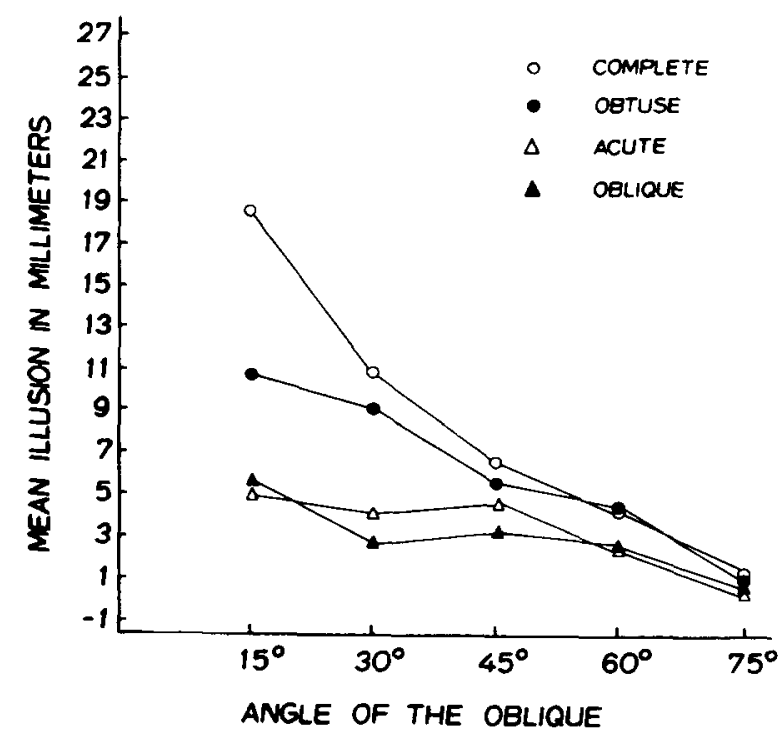

Figure 2. Mean illusion in millimeters plotted as a function of angle of interception of the oblique line for each of the complete, obtuse, acute, and oblique Poggendorff patterns. 


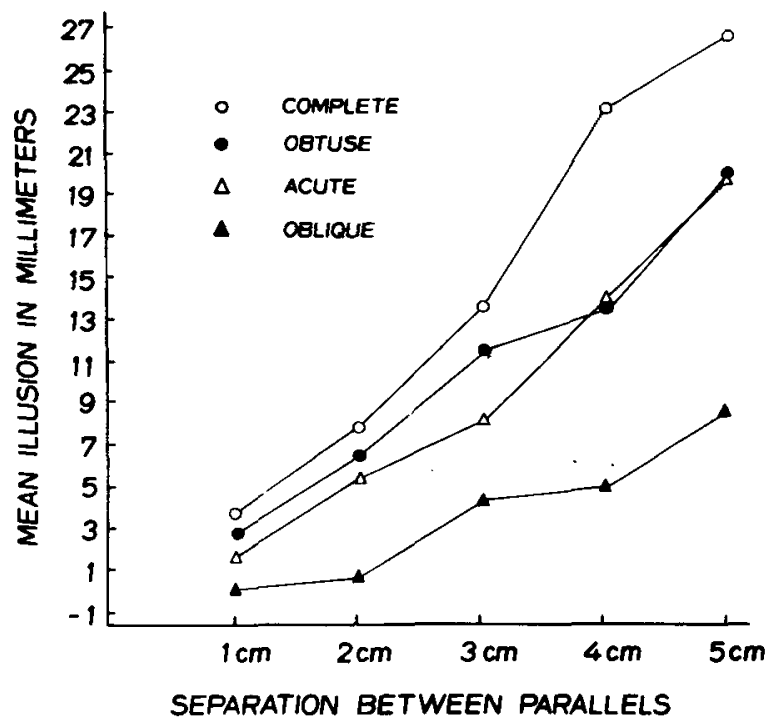

Figure 3. Mean illusion in millimeters plotted as a function of the separation of the parallel lines for each of the complete, obtuse, acute, and oblique Poggendorff patterns.

$[F(12,108)=7.70, p<.001]$. As seen in Figure 3, this interaction seems mainly attributable to the weaker effect of separation of the parallels on the oblique-only pattern. As for Experiment 1, the illusion scores for the four patterns were intercorrelated. These correlations had an average value of +0.67 and ranged from $+0.48(p<.10)$ to $+0.80 \quad(p<.005)$. The correlation of +0.48 (between the obtuse and the acute patterns) was the only one of the 12 computed in the two experiments that failed to reach significance at at least the .05 level.

The results of these two experiments provide a clear positive answer to each of the three questions phrased earlier. The three variants do produce the same illusory effect as the complete Poggendorff pattern; they do respond in the same way to manipulation of the two major variables that determine the magnitude of the illusory effect, angle of interception, and separation of the parallels; and, as the strong positive correlations among illusion scores on the different patterns show, individuals do respond in a consistent way to all four patterns.

Because of these findings, one is tempted to conclude that the different components represented in the three variants all arouse the same perceptual process as the complete Poggendorff, but to different degrees, and that this is the explanation of the consistently ordered magnitude of the illusory effect associated with the four patterns. One difficulty in the way of this conclusion is that while the patterns each contain a different component of the complete pattern, they also differ in terms of the total amount of the complete pattern which they contain. Thus, for example, the obtuse and the acute angle patterns differ in angular components, but the obtuse pattern contains more of the left-hand parallel as well. The greater illusion generated by the obtuse as compared to the acute pattern could as readily be attributed to the amount of pattern difference as to the angular difference. Experiment 3 was designed to examine the possibility that variation in illusion magnitude might depend on the total amount of illusion pattern present, independently of the presence of particular components.

\section{EXPERIMENT 3}

The obtuse angled pattern is the only variant suited to the purpose of this experiment, since the left-hand parallel can be systematically shortened, leaving the angle component intact. Lengthening the left-hand parallel in the acute angled pattern inevitably adds the obtuse angle and thus another component, rendering this variant unsuitable for an examination of the question of interest. Accordingly, the obtuse angle figure was chosen and the total amount of the pattern present was varied.

\section{Method}

Stimuli. The three versions of the obtuse-angle figure and the complete Poggendorff pattern used in this experiment are shown in Figure 4. As for Experiments 1 and 2, the patterns were duplicated on standard Ditto paper. In all patterns, the right-handed parallel was $18 \mathrm{~cm}$ long, the oblique line was $3.5 \mathrm{~cm}$ in length, and the obtuse angle was $150^{\circ}$. The separation of the parallels was fixed at $3 \mathrm{~cm}$. Three copies of each pattern were haphazardly arranged in a booklet with opaque blue pages interleaved, again, exactly as in the previous experiments.

Subjects. The subjects were nine men and two women undergraduate students, between the ages of 18 and 23 , who were paid for their participation.

Procedure. All details of procedure are exactly as for Experiments 1 and 2 .

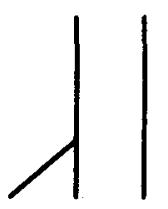

$\boldsymbol{A}$

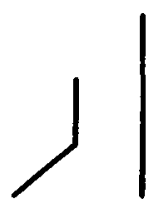

C

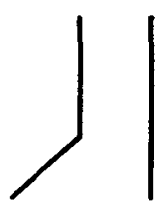

B

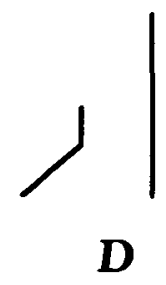

Figure 4. Patterns used in Experiment 3. In patterns B, $C$, and $D$, the left-hand parallel is progressively shortened. 


\section{Results and Discussion}

Analysis of variance of the data showed a significant effect of pattern $[F(3,30)=21.84, p<.001]$. Application of Tukey's H.S.D. test (Kirk, 1968) showed that the complete Poggendorff gave a larger illusion than any of the three incomplete variants and that these figures did not differ among themselves. The actual mean illusions (millimeters) associated with the patterns in the order in which they appear in Figure 4 are 17.0, 11.8, 12.0, and 12.7. This outcome provides some assurance that it is the particular components of the complete pattern that are contained in the variants that is important and not just the total amount of the pattern that is retained.

While the results of these experiments provide strong evidence that the complete Poggendorff and the three incomplete variants studied produce the same illusory effect, differing only in amount, they do not tell us much about the underlying perceptual process or processes. It is possible that all of the variants arouse a single process, but to varying degrees, or, alternatively, that the three incomplete patterns arouse different processes, each of which produces the same illusory effect, but of different magnitudes. If this latter were the case, then it would follow that the complete pattern arouses all three processes and that in combination they produce the large illusory effect that we observe.

Whatever the case, a single or multiple process, it is clear that its effectiveness in producing the illusion diminishes with practice (Cameron \& Steele, 1905; Coren \& Girgus, 1972; Pressey \& Sweeney, 1969). Indeed, Cameron and Steele report that after some 3,000 trials the illusion was virtually eliminated. It would seem possible to use this practice effect to examine the question of single or multiple processes, and such is intended in the experiment which follows.

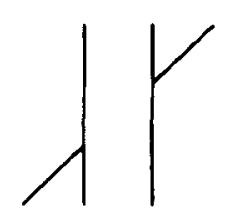

$\boldsymbol{A}$

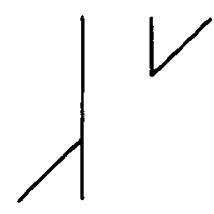

C

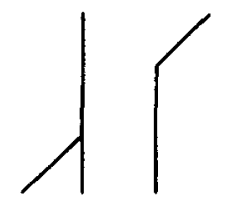

B

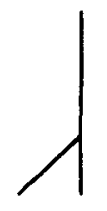

D
Figure 5. Patterns used in Experiment 4. (A) Complete, (B) obtuse, (C) acute, (D) oblique. Patterns $A$ and $D$ were also used in Experiment 5.

\section{EXPERIMENT 4}

The logic is this. Consider the hypothetical case of three processes, a different one for each of the obtuse-angled, acute-angled, and the oblique-only patterns that were used in Experiments 1 and 2 . Further, suppose that these three processes are all activated by the complete pattern. It would follow that practice effects obtained with all three should transfer positively to the complete pattern, the amount of transfer being related to the degree to which the particular process contributes to the total illusion. For example, practice with the obtuseangled pattern should reduce the illusion on the complete pattern when it is subsequently presented more than would be the case for the acute-angled pattern. This is because, as shown in Experiments 1 and 2 , the obtuse angled pattern produces a larger illusion than the acute-angled one and therefore the process it activates must contribute more to the total illusion. Reducing the effectiveness of this process by prior practice should eliminate or markedly reduce its contribution to the illusion when the complete pattern is presented. Another prediction required by this analysis is that practice on any one of the incomplete patterns should not transfer to any of the others since different processes are involved.

The alternative case, that of a single process that is aroused to a greater or lesser degree by the different patterns, leads to different predictions. It would still be expected that practice with the incomplete patterns would transfer positively to the complete pattern, but it would also be expected that practice with incomplete patterns would show positive transfer to one another.

\section{Method}

Stimuli. The patterns used in this experiment are shown in Figure 5. It will be noted that unlike the patterns used in the previously described experiments, here the left-hand parallel is intact in all patterns and the appropriate sections of the righthand parallel are removed to produce the variants. Again unlike the case in previous experiments, both oblique lines are present in all patterns. These stimuli were produced on white construction paper with black graphic tape, $1.6 \mathrm{~mm}$ in width. The parallel lines were $18 \mathrm{~cm}$ long and $3 \mathrm{~cm}$ apart. The obliques were $3.5 \mathrm{~cm}$ long and intercepted the parallels at a $45^{\circ}$ angle.

Apparatus. The apparatus consisted of a wooden frame in which two Plexiglas panels were affixed. The one on the right, from a front view, was immovable, while the one on the left could be moved freely up and down by a reversible electric motor that could be controlled either by the experimenter or the subject. The whole of the Poggendorff pattern, except for the left-hand oblique, was fastened to the immovable panel. The left-hand oblique was fixed to the movable panel, A $66-\mathrm{cm}$-wide and $51-\mathrm{cm}$-high piece of black bristol board was placed in front of the apparatus. It had an opening, $23 \mathrm{~cm}$ in width and $30 \mathrm{~cm}$ in height, cut from its center, through which the illusion pattern could be seen.

Subjects. The subjects were 20 men and 20 women undergraduate students, between the ages of 18 and 22 , who were paid for their participation. They were randomly assigned to one of four groups of 10 subjects each, with the constraint that there be 5 men and 5 women in each group. 
Procedure. Each of the four groups was given 50 trials of practice on one of the four patterns shown in Figure 5 and, after a 5 -min break while the pattern was changed, an additional 25 trials on the complete Poggendorff pattern.

The subjects were tested individually. When the subject arrived at the experimental room, he was seated at a table on which the apparatus was placed $1 \mathrm{~m}$ in front of him. A chinrest was provided. The subject was instructed that his task was to adjust the position of the left-hand oblique until it appeared collinear with the one on the right. He could move the oblique up or down, by depressing a button placed convenient to his preferred hand, whichever was appropriate given the presetting, until he was satisfied. On any given trial, he could move the oblique in one direction only.

Following the subject's setting, the experimenter read off the error of misalignment from a centimeter scale on the back of the apparatus. The experimenter then reset the position of the oblique to one of eight positions, four of which were different distances above the point of true collinearity and four of which were below. The subject then again made his setting. After 50 trials conducted in this manner, the subject was asked to wait in the hall for $5 \mathrm{~min}$ while, in all cases except where practice was on the complete pattern, the complete pattern was placed on the apparatus to replace the practiced variant. Those subjects who practiced on the complete pattern were also sent out into the hall for $5 \mathrm{~min}$ so that all subjects were treated in the same way. After $5 \mathrm{~min}$, the subject was brought back into the experimental room and performed 25 trials on the complete pattern.

\section{Results and Discussion}

The results for both practice and transfer trials are shown in Figure 6. A Lindquist (1953) Type I analysis of variance of the data for the practice trials in blocks of five showed a significant effect of pattern $[F(3,36)=11.97, p<.001]$, of trials $[\mathrm{F}(9,324=54.13, \mathrm{p}<.001]$, and a significant Pattern by Trials interaction $[F(27,324)=3.76$, $\mathrm{p}<.001]$. It will be noted that in terms of magnitude of illusion, the patterns in this experiment are ordered precisely as they were in Experiments 1 and 2 , providing assurance that the effects are not dependent on the details of the stimuli or on either psychophysical method or mode of presentation, all three of which were different in this experiment.

Analysis of variance of the transfer data showed that the pattern on which practice was carried out had no significant effect on subsequent performance on the complete pattern $[\mathrm{F}(3,36)=1.80$, n.s.]. Over the 25 transfer trials, there was a significant decrement $[F(4,12)=52.0, p<.001]$ but not a significant interaction $[\mathrm{F}(12,144)=0.60$, n.s.]. It appears, then, that practice on any one of the three illusion variants was equally effective in reducing the illusion on the subsequently presented complete pattern as was prior practice on the complete pattern itself.

To test to see if prior practice did significantly reduce the illusion, $t$ tests were done on the mean for the first five practice trials for the complete pattern and the means on the first five transfer trials for all four groups. In all cases $t$ ( 9 for complete, 18 for the 3 variants) $\geqslant 3.12, p<.05$.

Two features of the data warrant particular mention. The first is the substantial recovery of the

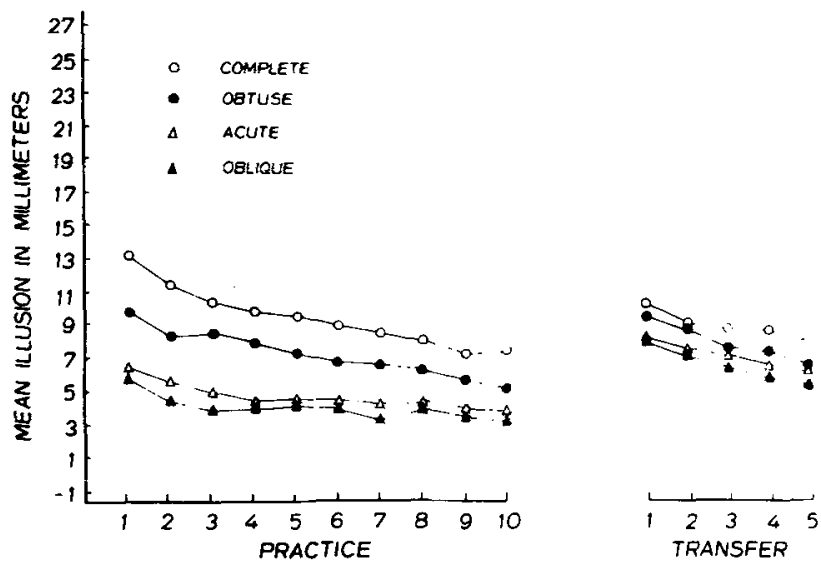

\section{TRIALS}

Figure 6. Practice data show mean illusion in millimeters plotted as a function of $\mathbf{1 0}$ blocks of five trials for each of the complete, obtuse, acute, and oblique patterns. Transfer data show mean illusion in millimeters plotted as a function of 5 blocks of five trials on the complete pattern following practice on each of the complete, obtuse, acute, and oblique patterns.

illusion during the 5-min break between the end of practice and the beginning of the transfer trials. To see if this recovery was significant, a t test was performed on the mean of the last block of five practice trials on the complete pattern and the first block of five transfer trials also on the complete pattern. The result was $t(9)=4.33, p<.001$. There is little to be said at the moment about this curious phenomenon, except that it clearly warrants further study in connection with the process involved in the decrement of the illusion with practice.

The second feature of the data worth special note concerns the transfer trials. While there is no significant effect of practice pattern on subsequent performance on the complete pattern, it is noteworthy that on the transfer trials the groups are ordered exactly opposite to what one might expect on the basis of a similarity explanation of transfer. Practice on the pattern identical to that used for transfer trials shows, in fact, the least transfer, while the oblique pattern, the most dissimilar to that used oni iransfer trials, shows the most transfer.

Singling out the practice data for the complete and oblique patterns for detailed scrutiny suggests the reason why the amounts of transfer associated with these two patterns were not more different than they are. It may be noted in Figure 6 that, for the complete pattern, the practice decrement proceeds regularly downward from the first to the last block of trials. For the oblique pattern, however, there is a substantial decrement from Trial Block 1 to Trial Block 3, but thereafter the illusory effect remains essentially stable at around $3.5 \mathrm{~mm}$. Whatever process brings about the decrement, then, appears to work continuously in the case of the 
complete pattern and brings the illusory effect progressively closer to the stable state obtained early on the oblique pattern. This suggests that if fewer practice trials were given with these two patterns, significantly different amounts of transfer might be observed. This prediction was tested in Experiment 5.

\section{EXPERIMENT 5}

\section{Method}

All details of stimuli and procedure for this experiment were identical to those of Experiment 4, except that only the complete and oblique patterns were used and there were 15 practice trials on each of these patterns followed by 50 transfer trials on the complete pattern.

Subjects. The subjects were 10 men and 10 women undergraduate students, between the ages of 18 and 31 , who were paid for their participation. They were randomly assigned to one of two experimental groups, with the constraint that there be 5 men and 5 women in each group.

\section{Results}

The results of this experiment are shown in Figure 7. Analysis of variance of the practice data showed a significant effect of pattern $[F(1,18)=53.48$, $\mathrm{p}<.001]$ and of trials $[\mathrm{F}(2,36)=23.97, \mathrm{p}<.001]$. The Pattern by Trials interaction did not reach significance $[F(2,36)=1.34$, n.s.]. This lack of a significant interaction suggests that the process responsible for the decrement acts equally for both patterns over these relatively few trials, although the levels of illusion associated with the two patterns are significantly different.

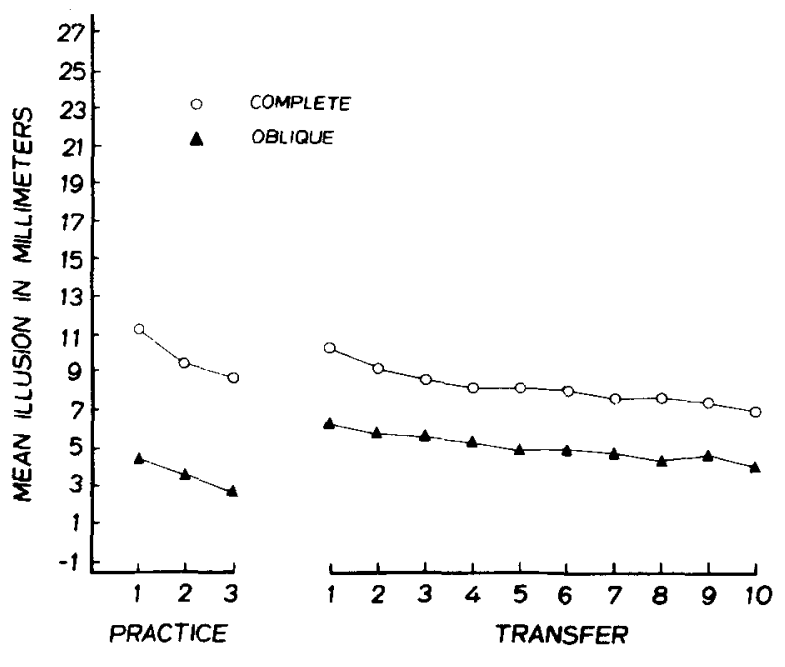

\section{TRIALS}

Figure 7. Practice data show mean illusion in millimeters plotted as a function of 3 blocks of five trials for each of the complete and oblique patterns. Transfer data show mean illusion in millimeters plotted as a function of 10 blocks of five trials on the complete pattern following practice on each of the complete and oblique patterns.
Analysis of variance of the transfer data show a significant effect of pattern $[F(1,18)=5.95, p<.05]$ and of trials $[F(9,162)=27.92, p<.001]$. The Pattern by Trials interaction did not approach significance $[F(9,162)=0.58, n . s$.$] and leads to the con-$ clusion that the advantage gained by practice on the oblique pattern was neither enhanced nor reduced over the long series of 50 transfer trials.

To see if the transfer observed was in fact significant, $t$ tests were done. The comparison of the mean of the first block of practice trials on the complete figure with the first blocks of transfer trials also on the complete figure (complete $\rightarrow$ complete) yielded a $t(9)=1.57$ (n.s.). The same comparison but with the first block on the complete figure following practice on the oblique pattern (oblique $\rightarrow$ complete) yielded a $\mathrm{t}(18)=4.3(\mathrm{p}<.001)$. With only 15 practice trials, then, significant transfer to the complete pattern is obtained only from the oblique pattern and not from the complete pattern itself.

\section{GENERAL DISCUSSION}

The weight of the evidence provided by these five experiments would appear to favor the conclusion that a single process underlies the Poggendorff illusion and its variants. Experiments 1 and 2 provide convincing evidence that removing parts of the complete pattern reduces the magnitude of the illusion, not by bringing different processes into play, but by rendering less effective the process that generates the large illusion with the complete pattern. As the data show, this process can be engaged more strongly with the Poggendorff variants, as with the complete pattern itself, by decreasing the angle at which the oblique intercepts the parallel or by increasing the separation of the parallels themselves. Supplementary support for a single-process explanation comes from the large and significantly positive correlations among illusion scores on the complete and incomplete patterns.

Were the data from Experiments 1 and 2 the only ones available, the case for a single process would be incompletely made. One could still argue, for example, that distinctive, but highly correlated, processes produce the illusory effects, say, for the acute angled figure and the obtuse angled figure, separate processes that are each more strongly engaged as angle of interception decreases or the separation of the parallels increases. But such an argument appears untenable in the face of the transfer data described in Experiment 4. It seems implausible that separate processes engaged during practice trials with the different patterns would evidence the effect of that practice to the same degree when the complete pattern was subsequently presented. This is especially so since such greatly different magnitudes of illusion are determined by 
the variants. The results of Experiment 5 underline the point. On the practice trials, the magnitude of illusion is significantly lower for the oblique pattern than for the complete pattern. And yet practice on the oblique pattern shows significant transfer to the subsequently presented complete pattern, while practice on the complete pattern itself does not. Incidentally, the outcomes of Experiments 4 and 5 would seem to obviate the need for the experiments concerned with transfer among the variants themselves that were suggested in the introduction to Experiment 4 as relevant to the single- or multipleprocess question. There now seems little reason to doubt that significant transfer effects would be obtained from practice on each variant to performance on the others, and such data would only provide additional supportive evidence in the case for a single underlying process, a case that seems already to be strongly made.

If one agrees that the data do support a single process explanation of the Poggendorff illusion, the problem becomes one of determining the nature of that process. A beginning to a solution may be to look first at the task which faces the subject when he attempts to assess collinearity in the Poggendorff display. Clearly, he has to extrapolate the oblique lines along a common perceptual path. To simplify the argument, let us suppose that what he does is to perceptually extend the lower left-hand oblique across the gap between the parallels. When he does this, he consistently comes out too low and so makes a directional error. The practice data show that this error is reduced over repeated attempts. But what is it that repeated attempts accomplish in order to bring about the reduced illusion?

An instructive observation in relation to this question is contained in Figure 6. Examination of the transfer data shows that those subjects who had prior practice on the complete figure fail, after 50 transfer trials still on the complete figure, to achieve as great an illusion reduction as is achieved after a mere 15 trials of practice on the oblique figure. Notice that on the transfer trials the "oblique subjects" begin with a lower illusion on the complete figure than characterizes the terminal performance on the transfer trials of those who had original practice on the complete figure. This observation makes it clear that the effectiveness of practice is not simply a matter of the number of trials, but also the pattern upon which practice is obtained.
Disappointingly, the present data do not permit us to say anything beyond that the subject perceives closer to true collinearity with the oblique pattern than with the complete pattern and that the effectiveness of prior exposure to the oblique pattern persists. The data also seem to support the view that the directionally consistent misperception of collinearity in all patterns may be attributed to a single underlying process, but they do not point to the nature of that process.

\section{REFERENCES}

Blix. M. Die sogenannte Poggendorff'sche optische Tauschung. Standinavisches A rchev für Physiologie. 1902, 13, 193-228.

Burmester, E. Beitrag zur experimentellen Bestimmung geometrisch-optischer Taeuschungen. Zeitschift für Psychologie und Physiologie de Sinnes, 1896, 12. 355-394.

Cameron, E. H., \& Steele, W. M. The Poggendorff illusion. Psychological Revien' Monograph Supplement, 1905, 7(1, Whole No. 29). 83-111.

COREN, S., \& Girgus, J. S. Illusion decrement in intersection line figures. Psychonomic Science, 1972, 26, 108-110.

DAY, R. H. The oblique line illusion: The Poggendorff effect without parallels. Quarterly Journal of Experimental Psychology, 1973, 25. 535-541. (a)

DAY, R. H. The Poggendorff illusion with obtuse and acute angles. Perception \& Psychophysics, 1973, 14, 590-590. (b)

Goldstein, M. B., \& Weintraub, D. J. The parallel-less Poggendorff: Virtual contours put the illusion down but not out. Perception \& Psychophysics, 1972. 11, 353-355.

GreEN, R. T., \& HoYle, E. M. The influence of spatial orientation on the Poggendorff illusion. Acta Psychologica, 1964, 22, 348-366.

KIRK, R. E. Experimental design: Procedures for the behavioral sciences. Belmont, Calif: Brooks/Cole, 1968.

Krantz, D. H., \& Weintraub, D. J. Factors affecting perceived orientation of the Poggendorff transversal. Perception \& Psychophysics, 1973, 14, $511-517$.

LINDQUiST, E. F. Design and analysis of experiments in psychology and education. Boston: Houghton Mifflin, 1953.

MYERS, J. L. Fundamentals of experimental design (2nd ed.). Boston: Allyn \& Bacon, 1972.

Pressey, A. W., \& SWeeney, O. A variation of the Poggendorff illusion. Perceptual \& Motor Skills, 1969, 28, 883-886.

Pressey, A. W., \& Sweeney, $O$. Acute angles and the Poggendorff illusion. Quarterly Journal of Experimental Psychology. 1972. 24, 169-174.

Restle, F. Illusions of bent lines. Perception \& Psychophysics, 1969, 5, 273-274

VELINSKY, P. ST. Explication physiologique de l'illusion de Poggendorff. Année Psychologique, 1925, 26, $107-116$.

Weintraus, D. J., \& KRantz. D. H. The Poggendorff illusion: Amputations, rotations, and other pertubations. Perception \& Psychophysics, 1971, 10, 257-263.

(Received for publication February 6, 1976; accepted October 22, 1976.) 